\title{
Vasorelaxation induced by common edible tropical plant extracts in isolated rat aorta and mesenteric vascular bed
}

\begin{abstract}
In this study, the vasodilatory actions of nine edible tropical plant extracts were investigated. Ipomoea batatas (sweet potato leaf), Piper betle (betel leaf), Anacardium occidentale (cashew leaf), Gynandropsis gynandra (maman leaf), Carica papaya (papaya leaf), and Mentha arvensis (mint leaf) extracts exhibited more than 50\% relaxing effect on aortic ring preparations, while Piper betle and Cymbopogon citratus (lemongrass stalk) showed comparable vasorelaxation on isolated perfused mesenteric artery preparation. The vascular effect on the aortic ring preparations were mainly endothelium-dependent, and mediated by nitric oxide (NO) as supported by the inhibition of action in the presence of N $\mathrm{r}$-nitro-1arginine (NOLA), an nitric oxide synthase (NOS) inhibitor, or by the removal of endothelium. In contrast, vasodilatory actions in resistance vessels (perfused mesenteric vascular beds) appear to involve several biochemical mediators, including NO, prostanoids, and endothelium-dependent hyperpolarizing factors (EDHFs). Total phenolic contents and antioxidant capacities varied among different extracts and found to be independent of vascular relaxation effects. This study demonstrates that many edible plants common in Asian diets to possess potential health benefits, affording protection at the vascular endothelium level.
\end{abstract}

Keyword: Vasorelaxation; Total phenol; Antioxidants; Endothelium-dependent relaxing factors; Endothelium-dependent hyperpolarizing factors; Blood vessels; Rat 